\title{
RESPOSTA DO FEIJOEIRO À ADUBAÇÃO FOSFATADA EM SOLOS DE CERRADO COM DIFERENTES HISTÓRICOS DE USO(1)
}

\author{
José Zilton Lopes Santos ${ }^{(2)}$, Antônio Eduardo Furtini Neto ${ }^{(3)}$, Álvaro \\ Vilela de Resende ${ }^{(4)}$, Leandro Flávio Carneiro ${ }^{(5)}$, Nilton Curi $^{(3)} \&$ \\ Bruno da Silva Moretti ${ }^{(6)}$
}

\begin{abstract}
RESUMO
Os diferentes tempos de cultivo e as diferentes formas de manejo podem influenciar na disponibilidade dos nutrientes, entre os quais o P. Objetivou-se com este estudo avaliar a resposta do feijoeiro à adubação com diferentes doses de $P$, em quatro Latossolos de Cerrado sob diferentes históricos de uso. As amostras dos Latossolos - (LVd1) textura argilosa, (LVd2) textura média alta, (LVAd1) textura média e (LVAd2) textura média baixa - foram coletadas na camada de 0 -20 $\mathrm{cm}$ de profundidade em locais cultivados há vários anos e, também, em áreas adjacentes não cultivadas (sob cerrado nativo). Com as amostras superficiais dos solos de cada área, foi conduzido um experimento em casa de vegetação, em delineamento inteiramente casualizado, num esquema fatorial $2 \times 4$ (dois históricos de uso cultivado e não cultivado, e quatro doses de $\mathrm{P}$ ), com quatro repetições. As doses de $P$, equivalentes a $0,120,240 \mathrm{e} 480 \mathrm{mg} \mathrm{dm}^{-3}$, foram aplicadas na forma de superfosfato triplo. A adição de doses crescentes de $\mathbf{P}$ aos solos promoveu aumento dos componentes relacionados ao crescimento do feijoeiro, principalmente matéria seca e conteúdo de $P$. A condição de solo já cultivado potencializou as respostas do feijoeiro à adubação, sobretudo na menor dose de $P$, e, nessa condição, houve maior absorção de $\mathrm{P}$ pelas plantas. $\mathrm{O}$ histórico de uso do solo influencia tanto a absorção quanto a eficiência de utilização de $P$ pelas plantas; no entanto, as condições que promovem maior absorção são opostas àquelas que permitem maior eficiência de utilização do nutriente, sobretudo nas condições de solo já cultivado. A
\end{abstract}

(1) Parte da Tese de Doutorado do primeiro autor apresentada ao Departamento de Ciência do Solo da Universidade Federal de Lavras - UFLA. Recebido para publicação em dezembro de 2009 e aprovado em outubro de 2010.

(2) Professor do Departamento de Engenharia Agrícola e Solos da Faculdade de Ciências Agrárias, Universidade Federal do Amazonas - UFAM. Av. General Rodrigo Otávio 3000, CEP 69077-000 Manaus (AM). E-mail: ziltonlopes@ufam.edu.br

(3) Professor do Departamento de Ciência do Solo, Universidade Federal de Lavras - UFLA. Caixa Postal 37, CEP 37200-000 Lavras (MG). E-mails: afurtini@ufla.br; niltcuri@ufla.br

(4) Pesquisador da Embrapa Milho e Sorgo. Caixa postal 285, CEP 35701-970 Sete Lagoas (MG). E-mail: Álvaro@cnpms.embrapa.br

(5) Professor Efetivo da Universidade Estadual de Mato Grosso do Sul - UEMS. Rodovia MS 306 km 6 Zona Rural - Unidade Universitária de Cassilândia. CEP: 79540-000. E-mail: lcarneiro@uems.br

(5) Mestrando do Departamento de Ciência do Solo, UFLA. E-mail: bsmoretti@hotmail.com 
disponibilidade de $\mathbf{P}$ no solo foi mais dependente das características físicas, químicas e mineralógicas do que do histórico de uso do solo.

Termos de indexação: manejo da adubação, utilização de fósforo, Phaseolus vulgaris.

\title{
ABSTRACT: BEAN PLANTS RESPONSE TO PHOSPHATE FERTILIZER IN CERRADO SOILS WITH DIFFERENT LAND USE HISTORY
}

\begin{abstract}
The different periods of tillage and forms of management may influence the availability of nutrients, including $P$. The objective of this study was evaluate the beans plants response to phosphate fertilizer in four Cerrado Latosols with different land use history. Samples of a clayey dystrophic Red Latosol (LVd1), a high medium texture Red Latosol (LVd2), a medium dystrophic Red-Yellow Latosol (LVAd1) and a low medium texture Red-Yellow Latosol (LVAd2) were collected in 0-20 $\mathrm{cm}$ depth layer in cultivated areas and surrounding uncultivated areas (native Cerrado). For each type of soil and concomitantly one experiment was carried out in greenhouse. A completely randomized design in a $2 \times 4$ factorial arrangement (two land use history-cultivated an uncultivated, and four $P$ doses) with four replication. The following $P$ doses: 0, 120, 240, and $480 \mathrm{mg} \mathrm{dm}^{-3}$ were used on the source triple superphosphate. The addition of increasing $P$ doses promoted increases in all components concerning growth of bean plants, mainly dry matter and P content. The formerly-cultivated condition improved the beans plants response to fertilization especially in the lower P dose and in this condition, a higher plant absorption was noticed. The land use history influence both absorption efficiency and $P$ use efficiency by plants. However, conditions that promote greater absorption are opposite those that allow more efficient use of nutrient, mainly in the formerly-cultivated condition. The available $P$ in the soil was more dependent on the physical, chemical and mineralogical characteristics than land use history.
\end{abstract}

Index terms: fertilization management, use of phosphorus, Phaseolus vulgaris.

\section{INTRODUÇÃO}

O P desempenha importante papel nas plantas, pois participa da constituição do ATP e de enzimas; quando em baixos teores no solo, a planta tem seu crescimento prejudicado (Kimani \& Derera, 2009). Em relação ao feijoeiro comum, esse nutriente tem proporcionado as maiores e mais frequentes respostas significativas no desenvolvimento dessa espécie, de modo que sua baixa disponibilidade no solo promove menor rendimento da cultura (Pastorini et al., 2000). Resposta do feijoeiro a doses maiores que $1.000 \mathrm{~kg} \mathrm{ha}^{-1}$ de $\mathrm{P}_{2} \mathrm{O}_{5}$ foi observada por Miranda et al. (2000) num Latossolo Vermelho-Escuro argiloso em condição de campo.

Entre os nutrientes, o P é aquele que frequentemente mais limita a produção das culturas nos solos da região de Cerrado (Carvalho et al., 1995). Isso ocorre pelo fato desse nutriente apresentar-se em formas pouco disponíveis aos vegetais e pelas características de elevada adsorção dos solos dessa região, fazendo com que a eficiência de fertilizantes fosfatados em sistemas de agricultura seja baixa, com somente 10 a $20 \%$ do nutriente sendo usado pelas culturas no ano de aplicação, e o valor residual raramente excede $50 \%$ (Bolland \& Gilkes, 1998).
Vários são os fatores que interferem na disponibilidade desse nutriente no solo, entre os quais podemse citar: o material de origem e o grau de intemperismo do solo (Novais \& Smith, 1999); textura, teor e qualidade da matéria orgânica do solo (MOS); e tipo de mineral. Embora apresente na maioria dos solos teores relativamente baixos, a MOS contribui, significativamente, na superfície específica e geração de cargas nos solos, graças ao seu alto grau de subdivisão. De modo semelhante, o tipo de mineral predominante na argila influencia, de maneira diferenciada, na superfície específica dos solos; a caulinita proporciona superfície específica de 10 a $30 \mathrm{~m}^{2} \mathrm{~g}^{-1}$, enquanto os óxidos de Fe apresentam superfície de 100 a $400 \mathrm{~m}^{2} \mathrm{~g}^{-1}$ (Novais \& Mello, 2007). Portanto, é de se esperar que solos tropicais, que apresentem diferentes proporções desses minerais e de MOS, tenham diferentes comportamentos quanto à superfície específica, o que provavelmente influenciará na adsorção de P no solo e disponibilidade deste para as plantas. Além desses fatores, a atividade biológica e vegetação (Santos et al., 2008) e, ainda, o manejo e o histórico de uso do solo também podem influenciar na disponibilidade desse nutriente e, consequentemente, na sua absorção pelas plantas (Wright, 2009). Considerando-se a expressiva influência da MOS na superfície específica dos solos tropicais, sistemas de uso que promovem 
maior aporte ou preservação da matéria orgânica no solo provavelmente contribuirão para maior alteração na geração de cargas e, consequentemente, alterarão a dinâmica de $\mathrm{P}$ no solo, além de promoverem aumento do compartimento orgânico de $\mathrm{P}(\mathrm{Po})$ (Matos et al., 2006). Em relação ao aumento do Po no solo, esse fato é bastante relevante, pois, de acordo com Condron \&Tiessen (2005), parece provável que a aquisição de $\mathrm{P}$ pelas plantas que crescem em solos altamente intemperizados, como a maioria dos solos tropicais, é regulada pela mineralização do Po; conforme Nziguheba \& Bunemann (2005), há evidências indiretas de que o Po em solos tropicais cultivados pode ser relativamente lábil, sendo bastante influenciado pelo histórico de uso do solo (Guggenberger et al., 1996).

Segundo Vincent et al. (2010), em solos minerais tropicais, em torno de $30 \%$ do $\mathrm{P}$ total está presente na forma orgânica e, dependendo da labilidade, esse estoque pode constituir-se numa importante fonte de P para as plantas. Xavier et al. (2010), avaliando as frações de $\mathrm{P}$ em diferentes agroecossistemas, verificaram que a distribuição desse nutriente nos compartimentos orgânicos e inorgânicos foi dependente das características específicas de cada agroecossistema e que a disponibilidade de $\mathrm{P}$ estava associada à ciclagem do seu compartimento orgânico. De modo semelhante, Wright (2009), avaliando os efeitos do uso da terra (cana-de-açúcar por 50 anos, pastagens por 100 anos e gramado por 60 anos) sobre a distribuição de $\mathrm{P}$ no perfil do solo e entre frações químicas para um histosol na região úmida da Flórida, verificou influência do histórico de uso nas frações de $\mathrm{P}$ ligado a Fe, $\mathrm{Al}$ e $\mathrm{Ca}$, além dos compartimentos inorgânicos e orgânicos de P. Por outro lado, Chapuis-Lardy et al. (2001), estudando o status de P orgânico em Latossolos de ambientes fisionomicamente diferentes no cerrado, verificaram que a vegetação que proporcionava maior entrada de matéria orgânica continha mais Po na forma lábil do que as demais. No entanto, o Po foi pouco influenciado pelo histórico de uso do solo, exceto na profundidade de $0-10 \mathrm{~cm}$. Dessa forma, esses resultados mostram que a compreensão das mudanças na disponibilidade de $\mathrm{P}$ em função da mineralogia, manejo e histórico de uso do solo pode contribuir para o desenvolvimento de estratégias de manejo que proporcionarão melhora na aquisição de $\mathrm{P}$ pela planta e, por conseguinte, maior produtividade nos solos tropicais.

No presente trabalho, objetivou-se avaliar, em casa de vegetação, a resposta do feijoeiro a doses de $\mathrm{P}$ em quatro Latossolos de Cerrado com diferentes históricos de uso.

\section{MATERIAL E MÉTODOS}

O trabalho foi conduzido no Departamento de Ciência do Solo da Universidade Federal de Lavras no período de maio a agosto de 2007, utilizando-se amostras de um Latossolo Vermelho distrófico (LVd1) textura argilosa, um Latossolo Vermelho distrófico (LVd2) textura média alta, um Latossolo VermelhoAmarelo distrófico (LVAd1) textura média e um Latossolo Vermelho-Amarelo distrófico (LVAd2) textura média baixa. Para cada um dos solos coletados na Fazenda Alto Alegre (Planaltina de Goiás-GO), foram obtidas amostras da camada de $0-20 \mathrm{~cm}$, em locais cultivados há vários anos (com calagem e adubações fosfatadas periódicas) e em áreas adjacentes não cultivadas (sob Cerrado aberto baixo uniforme, exceto ao solo LVd2, onde predominava uma transição de cerrado para mata de galeria).

O LVd1 e o LVAd1 apresentavam-se sob o sistema plantio direto (SPD) há mais de 10 anos. Nesse período, foi feito um preparo com arado de aiveca no primeiro solo e uma subsolagem no segundo, de forma que, à época da coleta, estavam com seis e quatro anos de plantio direto contínuo, respectivamente. Essas duas áreas vinham sendo cultivadas com soja e milho em sistema de sucessão e recebendo adubação fosfatada média anual de $80 \mathrm{~kg} \mathrm{ha}^{-1}$ de $\mathrm{P}_{2} \mathrm{O}_{5}$. Em 2000/2001, o LVd1 recebeu, também, uma adubação corretiva com $650 \mathrm{~kg} \mathrm{ha}{ }^{-1}$ de fosfato reativo de Gafsa $\left(28 \%\right.$ de $\mathrm{P}_{2} \mathrm{O}_{5}$ total).

O LVd2 e o LVAd2 foram usados com pastagem de capim-braquiária (Brachiaria brizantha, cultivar Marandu) de 1986 a 1999, cultivados com soja e milho por cinco safras (antes da última foi feita uma subsolagem), e, novamente, braquiária por três anos. Para as culturas anuais, foram fornecidos, em média, $88 \mathrm{~kg} \mathrm{ha}^{-1} \mathrm{ano}^{-1} \mathrm{de}_{2} \mathrm{O}_{5}$. Em 1999/2000, os dois solos receberam adubação corretiva com $650 \mathrm{~kg} \mathrm{ha}^{-1}$ de fosfato reativo de Gafsa. Antes da instalação dos experimentos, os solos foram analisados, sendo os atributos químicos, físicos e mineralógicos apresentados no quadro 1.

Foi instalado um experimento com cada solo concomitantemente, em vasos de polietileno com capacidade para $4,0 \mathrm{dm}^{3}$. Os solos receberam carbonato de cálcio e carbonato de magnésio puro para análise (p.a.), na relação de quatro partes de Ca para uma de $\mathrm{Mg}$, objetivando elevar a saturação por bases a $70 \%$ (exceto os solos LVAd1 e LVd2 na condição cultivada, que não necessitaram de calagem). Em seguida, os solos foram incubados por 30 dias.

Para cada experimento, os tratamentos foram dispostos em delineamento inteiramente casualizado, num esquema fatorial $2 \times 4$ (duas condições de histórico de uso do solo e quatro doses de P) com quatro repetições. Os tratamentos foram constituídos por duas condições de histórico de uso do solo (não cultivado ou cultivado) e quatro doses de $\mathrm{P}(0,120$, 240 e $480 \mathrm{mg} \mathrm{dm}^{-3} \mathrm{de} \mathrm{P}$ ), na fonte superfosfato triplo. $\mathrm{O}$ fornecimento das doses de $\mathrm{P}$ foi efetuado com base no teor de $\mathrm{P}_{2} \mathrm{O}_{5}$ total da fonte $(46,1 \%)$.

Em todas as parcelas, efetuou-se uma adubação básica com macro e micronutrientes, constituída de: 100 mg de N; 100 mg de K nos solos não cultivados e 
Quadro 1. Atributos químicos, físicos e mineralógicos dos solos utilizados no experimento, antes da aplicação dos tratamentos

\begin{tabular}{|c|c|c|c|c|c|c|c|c|}
\hline \multirow{3}{*}{ Atributo do solo } & \multicolumn{8}{|c|}{ Solo } \\
\hline & \multicolumn{2}{|c|}{ LVd1 } & \multicolumn{2}{|c|}{ LVd2 } & \multicolumn{2}{|c|}{ LVAd1 } & \multicolumn{2}{|c|}{ LVAd2 } \\
\hline & $\mathrm{NC}$ & $\mathbf{C}$ & $\mathrm{NC}$ & $\mathbf{C}$ & $\mathrm{NC}$ & $\mathbf{C}$ & NC & $\mathbf{C}$ \\
\hline $\mathrm{pH}\left(\mathrm{H}_{2} \mathrm{O}\right)$ & 5,5 & 5,7 & 4,9 & 7,0 & 5,2 & 5,8 & 5,2 & 5,7 \\
\hline P Mehlich $1\left(\mathrm{mg} \mathrm{dm}^{-3}\right)$ & 1,0 & 11,6 & 1,9 & 16,9 & 1,5 & 26,5 & 1,7 & 11,9 \\
\hline P Resina $\left(\mathrm{mg} \mathrm{dm}^{-3}\right)$ & 3,6 & 37,0 & 3,2 & 13,2 & 2,8 & 35,3 & 3,1 & 12,9 \\
\hline $\mathrm{K}\left(\mathrm{mg} \mathrm{dm}^{-3}\right)$ & 43 & 123 & 109 & 94 & 58 & 245 & 47 & 156 \\
\hline $\mathrm{Ca}\left(\mathrm{cmol}_{\mathrm{c}} \mathrm{dm}^{-3}\right)$ & 0,5 & 2,2 & 0,7 & 4,4 & 0,5 & 3,6 & 0,5 & 2,1 \\
\hline $\mathrm{Mg}\left(\mathrm{cmol}_{\mathrm{c}} \mathrm{dm}^{-3}\right)$ & 0,2 & 0,8 & 0,2 & 2,2 & 0,2 & 1,4 & 0,2 & 0,7 \\
\hline $\mathrm{Al}\left(\mathrm{cmol}_{\mathrm{c}} \mathrm{dm}^{-3}\right)$ & 0,8 & 0,2 & 1,6 & 0,0 & 1,9 & 0,1 & 1,6 & 0,3 \\
\hline $\mathrm{H}+\mathrm{Al}\left(\mathrm{cmol}_{\mathrm{c}} \mathrm{dm}^{-3}\right)$ & 6,5 & 4,0 & 6,3 & 1,3 & 6,5 & 2,6 & 6,8 & 3,3 \\
\hline $\mathrm{SB}\left(\mathrm{cmol}_{\mathrm{c}} \mathrm{dm}^{-3}\right)$ & 0,8 & 3,4 & 1,2 & 6,8 & 0,8 & 5,6 & 0,9 & 3,1 \\
\hline $\mathrm{t}\left(\mathrm{cmol}_{\mathrm{c}} \mathrm{dm}^{-3}\right)$ & 1,7 & 3,6 & 2,8 & 6,8 & 2,7 & 5,6 & 2,5 & 3,4 \\
\hline $\mathrm{T}\left(\mathrm{cmol}_{\mathrm{c}} \mathrm{dm}^{-3}\right)$ & 7,4 & 7,4 & 7,5 & 8,1 & 7,3 & 8,2 & 7,5 & 6,5 \\
\hline $\mathrm{V}(\%)$ & 11,4 & 45,6 & 16,2 & 84,0 & 11,0 & 68,3 & 7,7 & 48,2 \\
\hline m (\%) & 49,7 & 5,7 & 57,3 & 0,0 & 70,0 & 0,0 & 64,5 & 8,7 \\
\hline S-sulfato $\left(\mathrm{mg} \mathrm{dm}^{-3}\right)$ & 5,4 & 17,4 & 3,7 & 4,6 & 8,9 & 10,1 & 5,9 & 9,8 \\
\hline $\mathrm{B}\left(\mathrm{mg} \mathrm{dm}^{-3}\right)$ & 0,2 & 0,2 & 0,3 & 0,3 & 0,1 & 0,2 & 0,3 & 0,3 \\
\hline $\mathrm{Cu}\left(\mathrm{mg} \mathrm{dm}^{-3}\right)$ & 0,5 & 2,0 & 0,3 & 0,6 & 0,6 & 0,5 & 0,1 & 1,1 \\
\hline $\mathrm{Fe}\left(\mathrm{mg} \mathrm{dm}^{-3}\right)$ & 27,2 & 26,9 & 44,1 & 22,6 & 86,2 & 46,7 & 73,6 & 98,5 \\
\hline $\operatorname{Mn}\left(\mathrm{mg} \mathrm{dm}^{-3}\right)$ & 3,4 & 7,5 & 3,7 & 7,5 & 1,2 & 8,6 & 3,6 & 4,5 \\
\hline $\mathrm{Zn}\left(\mathrm{mg} \mathrm{dm}^{-3}\right)$ & 0,2 & 4,1 & 0,3 & 1,1 & 0,3 & 2,0 & 0,3 & 1,1 \\
\hline $\mathrm{P}$ remanescente $\left(\mathrm{mg} \mathrm{L}^{-1}\right)$ & 9,8 & 14,6 & 15,4 & 19,9 & 23,2 & 36,0 & 26,7 & 32,6 \\
\hline Matéria orgânica $\left(\mathrm{g} \mathrm{kg}^{-1}\right)$ & 32,0 & 28,0 & 25,0 & 25,0 & 21,0 & 27,0 & 25,0 & 25,0 \\
\hline Areia $\left(\mathrm{g} \mathrm{kg}^{-1}\right)$ & 104 & 126 & 380 & 417 & 587 & 464 & 543 & 500 \\
\hline Silte $\left(\mathrm{g} \mathrm{kg}^{-1}\right)$ & 326 & 304 & 300 & 253 & 213 & 326 & 327 & 350 \\
\hline Argila $\left(\mathrm{g} \mathrm{kg}^{-1}\right)$ & 570 & 570 & 320 & 330 & 200 & 210 & 130 & 150 \\
\hline $\mathrm{Ct}\left(\mathrm{g} \mathrm{kg}^{-1}\right)$ & 203,1 & 242,0 & 78,7 & 88,7 & 28,0 & 35,2 & 17,0 & 21,0 \\
\hline $\mathrm{Gb}\left(\mathrm{g} \mathrm{kg}^{-1}\right)$ & 115,9 & 111,7 & 11,4 & 7,0 & 7,6 & 5,3 & 1,9 & 4,3 \\
\hline Hematita $\left(\mathrm{g} \mathrm{kg}^{-1}\right)$ & 4,7 & 4,7 & 2,6 & 2,7 & 0,9 & 1,0 & 0,6 & 0,7 \\
\hline Goethita $\left(\mathrm{g} \mathrm{kg}^{-1}\right)$ & 35,1 & 34,1 & 5,2 & 5,7 & 2,4 & 3,7 & 1,1 & 1,9 \\
\hline
\end{tabular}

LVd1: Latossolo Vermelho textura argilosa, LVd2: Latossolo Vermelho textura média alta, LVAd1: Latossolo Vermelho-Amarelo distrófico textura média e LVAd2: Latossolo Vermelho-Amarelo distrófico textura média baixa.

$30 \mathrm{mg}$ nos solos cultivados; $40 \mathrm{mg}$ de $\mathrm{S} ; 0,8 \mathrm{mg}$ de B; 1,5 mg de $\mathrm{Cu} ; 3,6 \mathrm{mg}$ de $\mathrm{Mn} ; 5,0 \mathrm{mg}$ de Zn; e 0,15 mg de Mo por $\mathrm{dm}^{3}$ de solo. Essa adubação foi feita com reagentes p.a., na forma de solução, e após o seu fornecimento o solo foi novamente incubado por 20 dias.

Após o período de incubação, procedeu-se à semeadura de seis sementes de feijão por vaso e, 12 dias após plantio (DAP), foram efetuados desbastes, deixando-se apenas três plantas por vaso. Durante a incubação e a condução do experimento, a umidade foi mantida em $60 \%$ do volume total de poros, por meio de pesagem diária dos vasos e adição de água deionizada. Adubações de cobertura com N e K foram realizadas de acordo com o crescimento das plantas. Os tratamentos conduzidos nos solos cultivados receberam $300 \mathrm{mg}$ de $\mathrm{N}$ e $150 \mathrm{mg}$ de $\mathrm{K}$ por $\mathrm{dm}^{3}$, ao passo que os solos não cultivados receberam $300 \mathrm{mg}$ de $\mathrm{N}$ e $300 \mathrm{mg}$ de $\mathrm{K}$ por $\mathrm{dm}^{3}$, parcelados em três aplicações.

No início do florescimento do feijoeiro (38 DAP), foi colhida a parte aérea de uma planta de cada parcela.
As plantas colhidas foram secas em estufa de circulação forçada de ar, à temperatura de $60{ }^{\circ} \mathrm{C}$, sendo, posteriormente, trituradas e submetidas à análise química, para determinação dos teores totais de P (Malavolta et al., 1997). Com base nos teores de $\mathrm{P}$ e na produção de matéria seca da parte aérea (MSPA), foi calculado o acúmulo do nutriente na parte aérea. A eficiência de absorção de $\mathrm{P}$ foi estimada conforme a expressão: [(P absorvido na dose aplicada - P absorvido na testemunha) / dose aplicada] x 100 (Baligar et al., 1990).

As duas plantas restantes foram cultivadas até o final do ciclo (75 DAP), ocasião em que a parte aérea foi colhida. Caules e vagens foram secos em estufa, juntamente com as folhas que senesceram e caíram ao longo do ciclo da cultura, a fim de determinar a produção de MSPA. Os grãos foram secos em estufa e triturados, para determinação do teor de P. A eficiência de utilização de $\mathrm{P}$ (EUP) para grãos foi obtida de acordo com a expressão: $\mathrm{EUP}=[$ (matéria seca de grãos) $)^{2}$ / (P acumulado nos grãos)] (Siddiqi \& Glass, 1981). 
Os dados obtidos foram submetidos à análise de variância. Foram ajustados modelos de regressão para a produção de matéria seca da parte aérea e conteúdo de $\mathrm{P}$ na parte aérea no florescimento, além de eficiência de absorção no florescimento e utilização de P para grãos, como variáveis dependentes das doses de P.

\section{RESULTADOS E DISCUSSÃO}

\section{Crescimento e absorção de $\mathbf{P}$}

A produção de MSPA obtida no final do ciclo das plantas aumentou de forma linear com a adição de $\mathrm{P}$ em todos os solos, sendo influenciada também pelo histórico de uso (Figura 1a, c, d), exceto o solo LVd2 (Figura 1b), em que a resposta foi quadrática, tendendo a estabilizar a partir da terceira dose e não sendo alterada pelo histórico de uso do solo. Esses resultados mostram que a resposta da planta à adubação fosfatada depende, entre outros fatores, da disponibilidade de $\mathrm{P}$ no solo, o que é alterado pelo histórico de uso; no entanto, quando não se adiciona $\mathrm{P}$ nesses solos, as produções são muito pequenas ou quase nulas. Por outro lado, a quantidade de P fornecida sobrepujou os fatores de solo quanto à influência na resposta das plantas à adubação. Contudo, o grau de limitação das omissões de P (dose zero) variou entre os solos, assim como a resposta ao fornecimento desse nutriente. Nota-se que o solo LVd1, que apresenta um histórico de uso e um manejo bastante semelhante ao do LVAd1, apesar de o primeiro estar há mais tempo no SPD e ainda ter recebido adição extra de $\mathrm{P}$ como fosfato reativo de Gafsa, fatores que contribuem para maior disponibilidade de P (Santos et al., 2003), não promoveram maior produção de MSPA em relação ao segundo solo. Esse comportamento está possivelmente relacionado às características intrínsecas do solo, que favorecem uma maior fixação e menor disponibilidade de $\mathrm{P}$ para as plantas, como maior teor de argila e dos minerais caulinita, gibbsita, hematita e goethita no solo LVd1 em relação ao LVAd2 (Quadro 1). Além disso, verifica-se que, apesar desses solos cultivados estarem sob SPD, os teores de matéria orgânica (Quadro 1) encontrados neles enquadram-se na classe média (CFSEMG, 1999). Dessa forma, é provável que os efeitos de radicais orgânicos, que ocupariam sítios de adsorção de $\mathrm{P}$, reduzindo a capacidade de retenção desse nutriente pelo solo (Sá, 2004), sejam menos intensos, o que provavelmente contribui para maior expressão dos efeitos da mineralogia e textura dos solos.

Os maiores incrementos de MSPA para cada unidade de $\mathrm{P}$ adicionada foram verificados na condição de solo não cultivado, como mostram os coeficientes angulares dos modelos (Figura 1a, c, d), o que contribuiu para a tendência de equiparação ao crescimento observado nas duas condições de cultivo do solo à medida que as doses aplicadas são aumentadas nos solos LVd1 e LVAd1. Esse comportamento confirma as informações de Raij (1991), de que os efeitos da adubação fosfatada nas culturas são mais visíveis em solos de baixa fertilidade. No entanto, a condição de solo cultivado proporcionou maior produção de MSPA em relação à não cultivada, sobretudo na ausência de adubação fosfatada e na dose baixa de $\mathrm{P}\left(120 \mathrm{mg} \mathrm{dm}^{-3}\right)$, exceto o solo LVd2 (Figura 1b), que não apresentou efeito do histórico de uso. No caso do LVd2, esse efeito possivelmente está relacionado aos altos valores de $\mathrm{pH}$, dos teores de Ca, $\mathrm{Mg}$ e da V (Quadro 1) apresentados por esse solo, e esses atributos influenciam na disponibilidade de P. Esses altos valores podem ser em razão de o solo LVd2 estar localizado num ponto de cota inferior em relação ao solo LVAd2, fato que poderia contribuir para maior recebimento tanto de sedimentos ou até mesmo de insumos, ao longo dos anos, oriundos dos pontos de cotas mais elevadas.

O padrão de resposta do feijoeiro quanto ao acúmulo de $\mathrm{P}$ no florescimento, dado pelo conteúdo do nutriente na parte aérea (CPPA), também foi dependente das doses de $\mathrm{P}$ e do histórico de uso dos solos (Figura 1e, f, $\mathrm{g}, \mathrm{h})$. O aumento das doses promoveu maior disponibilidade de $\mathrm{P}$ e, consequentemente, maior absorção e incorporação desse nutriente na biomassa. Houve gradativo acúmulo de $\mathrm{P}$ com as doses fornecidas e destacado efeito do histórico de uso, especialmente nos dois solos LVA que apresentam teores de argila semelhantes (Quadro 1) e históricos de uso diferentes (Figura 1g, h), para os quais os cultivos e adubações anteriores favoreceram uma maior absorção e acúmulo de $\mathrm{P}$ pela parte aérea. No entanto, para o LVAd1 essa maior absorção de $\mathrm{P}$, principalmente nas maiores doses na condição cultivada (Figura 1g), não foi traduzida em maior produção de MSPA (Figura 1c), quando comparada à condição não cultivada. Isso deve estar relacionado com uma alta disponibilidade de $\mathrm{P}$ $\left(26,5 \mathrm{mg} \mathrm{dm}^{-3}\right)$ nesse solo (Quadro 1), indicando que nessa condição de recebimento de $\mathrm{P}$ em adubações anteriores a quantidade de $\mathrm{P}$ absorvida foi suficiente para que a planta atingisse sua capacidade metabólica plena, e uma quantidade expressiva do $\mathrm{P}$ absorvido constituiu-se apenas em consumo de luxo, visto que a EUP (Figura 2g) manteve-se constante com a adição de doses crescentes do nutriente na condição cultivada.

No solo LVd2 que recebeu menor adição de $\mathrm{P}$ ao longo dos anos de cultivos (apenas cinco anos de sucessão soja/milho), quando comparado ao solo LVAd1 (com 10 anos de sucessão soja/milho), observou-se menor acúmulo de $\mathrm{P}$ na parte aérea em relação a este último, apesar de os dois pertencerem à mesma classe textural. Isso indica que, para alguns solos, uma maior adição de $\mathrm{P}$ em cultivos anteriores pode permitir amortização nas doses da adubação fosfatada em cultivos futuros. Esses resultados corroboram aqueles encontrados por Wright (2009), indicando que a disponibilidade de $\mathrm{P}$ é dependente tanto do histórico de uso quanto do manejo do solo. Na condição não cultivada, a adição de doses crescentes de $\mathrm{P}$ promoveu 

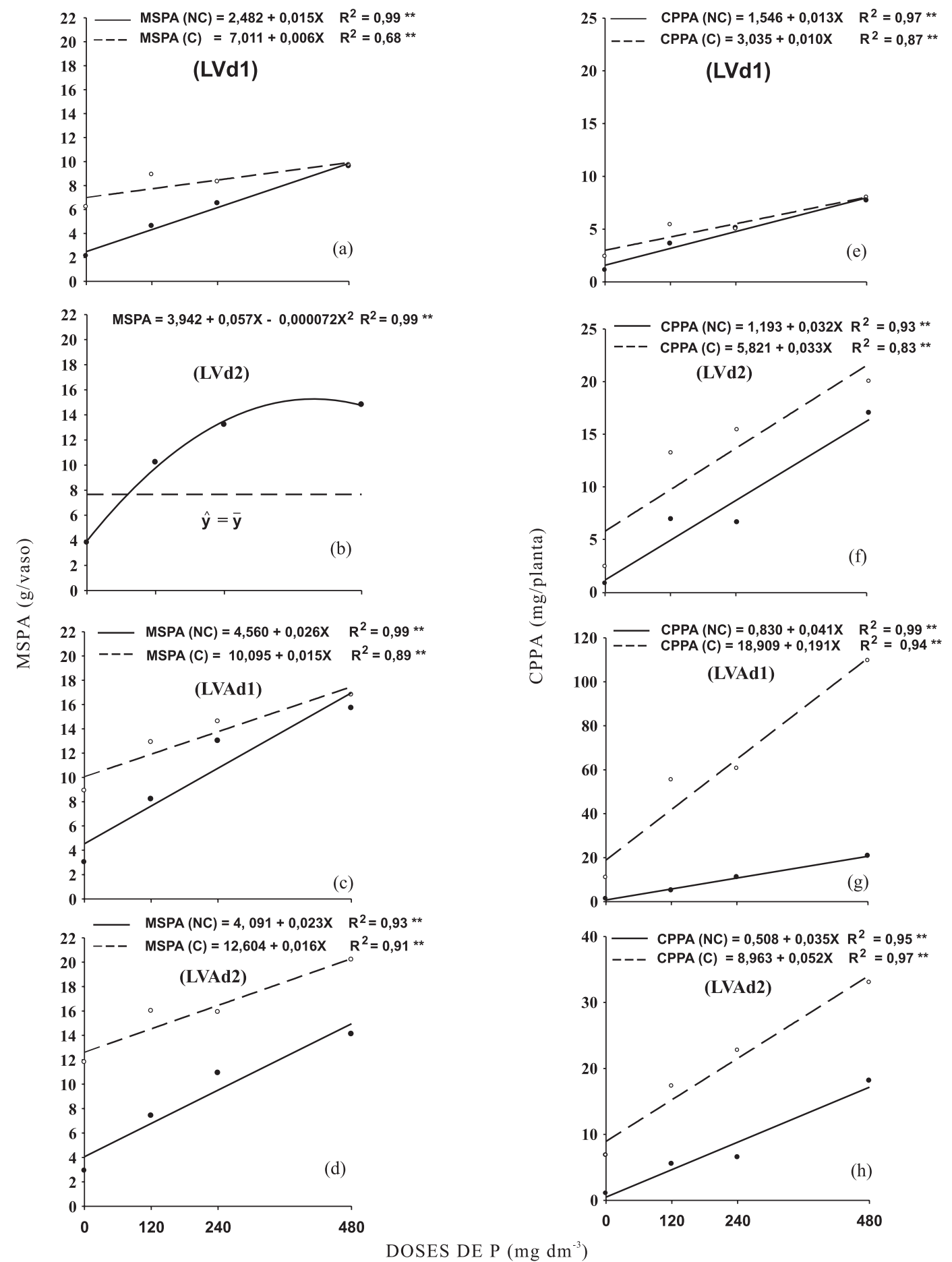

Figura 1. Produção de matéria seca da parte aérea no final do ciclo (MSPA) (a, b, c e d); conteúdo de fósforo na parte aérea no florescimento (CPPA) (e, f, g e h) nos solos LVd1, LVd2, LVAd1 e LVAd2, em função das doses de $P$, na condição de solo não cultivado (NC) e cultivado (C).

valores de acúmulos semelhantes entre os diferentes solos, exceto o LVd1, que proporcionou o menor acúmulo nessa condição. Esse fato possivelmente está relacionado às características intrínsecas deste solo, contribuindo para maior fixação de $\mathrm{P}$. O contrário ocorreu quando doses crescentes foram adicionadas na condição de solo cultivado, mostrando que o histórico de uso do solo teve importante participação nesse comportamento. É importante ressaltar que a influência do histórico de uso no acúmulo de P está relacionada com as características mineralógicas e texturais do solo, de modo que a textura menos argilosa dos solos LVA pode, também, ter contribuído para um padrão de resposta diferente em relação aos solos LV. 

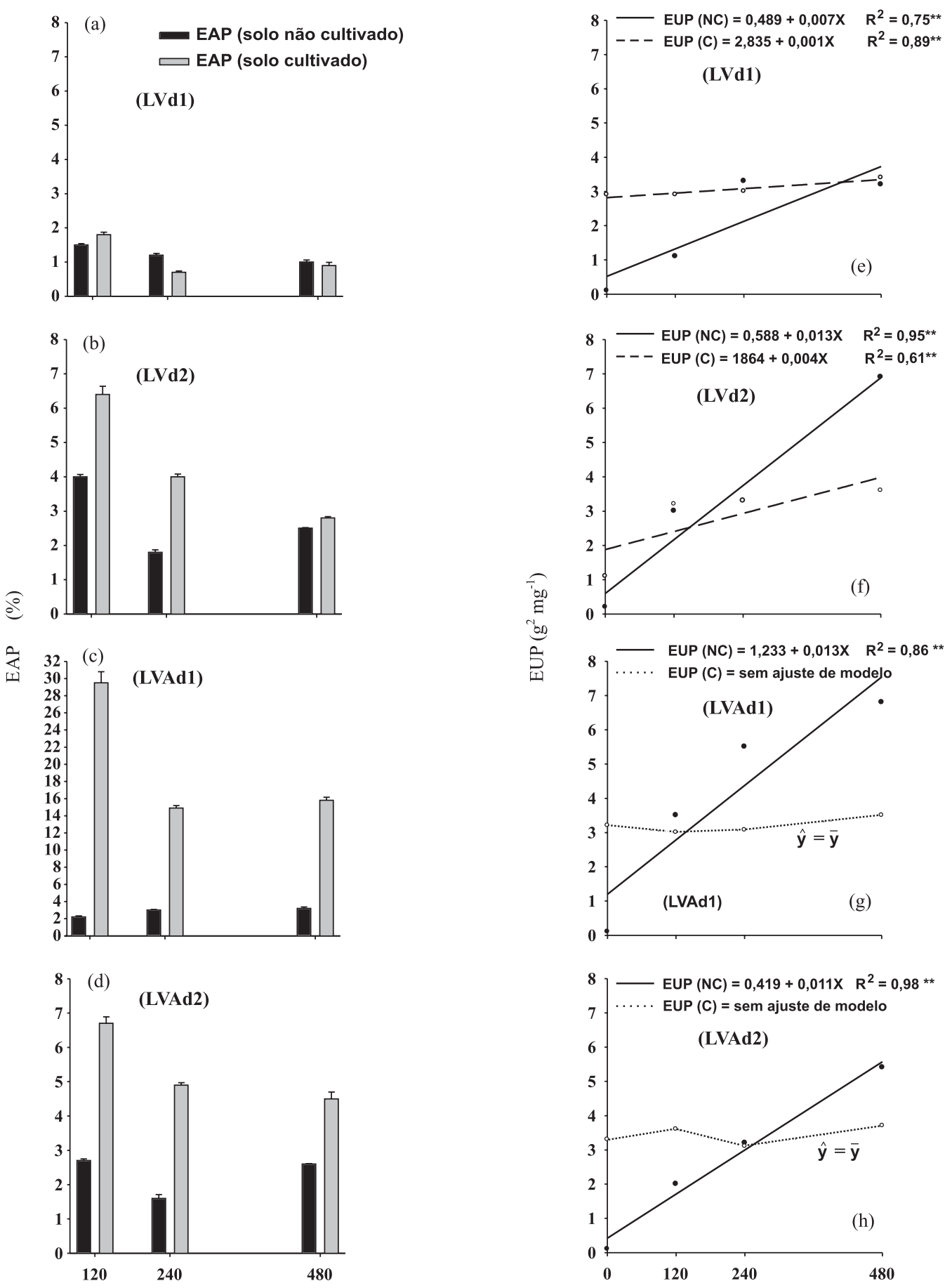

DOSES DE P $\left(\mathrm{mg} \mathrm{dm}^{-3}\right)$

Figura 2. Eficiência de absorção de fósforo (EAP) (a, b, c e d) e eficiência de utilização de fósforo para grãos (EUP) (e, f, g e h) nos solos LVd1, LVd2, LVAd1 e LVAd2, em função das doses de P, na condição de solo não cultivado $(\mathrm{NC})$ e cultivado $(\mathrm{C})$.

O maior acúmulo de $\mathrm{P}$ na parte aérea, quando as plantas cresceram em solos já cultivados (Figura 1e, $\mathrm{f}, \mathrm{g}, \mathrm{h})$, é um aspecto agronomicamente relevante, por indicar que há alguma saturação dos sítios de adsorção de $\mathrm{P}$ no solo, de forma que uma porção mais expressiva do nutriente fornecido na adubação subsequente é incorporada pelo vegetal. Considerando que nos sistemas de cultivos utilizados há pouca exportação da matéria seca, é plausível afirmar que o $\mathrm{P}$ disponível nesses solos cultivados é regulado também pela mineralização do Po, pois há evidências indiretas de que o Po em solos tropicais cultivados pode ser relativamente lábil (Nziguheba \& Bunemann, 2005). 


\section{Eficiências de absorção e de utilização de $\mathbf{P}$}

A eficiência de absorção de $\mathrm{P}$ (EAP) pelo feijoeiro, que se refere à percentagem do $\mathrm{P}$ aplicado que foi absorvida e acumulada na MSPA no florescimento, foi alterada pelas doses crescentes de $\mathrm{P}$ e pelo histórico de uso dos solos (Figura 2a, b, c, d). Os maiores valores de EAP foram observados para as menores doses de $\mathrm{P}$, havendo diminuição da EAP com o aumento da dose fornecida, principalmente nos solos já cultivados - fatos que devem estar ligados tanto ao fornecimento de fertilizantes fosfatados quanto a uma maior predominância de P na forma orgânica lábil. Esses resultados mostram que adubos fosfatados adicionados ao solo apresentam, além do efeito imediato sobre a cultura que se segue à adubação, efeito residual de alguns anos em função de vários fatores, como tipo de solo, fonte, dose e método de aplicação do fertilizante, sistema de preparo e sequência dos cultivos (Santos et al., 2008). Os dados demonstram também que a capacidade de absorção pela planta não foi proporcional à dose aplicada, sugerindo que nos cultivos em solos anteriormente adubados a eficiência de absorção tende a decrescer à medida que ocorre o aumento das doses do fertilizante - comportamento que não fica nítido para solos que não foram adubados anteriormente, indicando que o histórico de uso das áreas pode interferir nessa eficiência.

Nota-se que o comportamento da EAP é inverso ao observado para o acúmulo de $\mathrm{P}$ na parte aérea (Figura 1e,f, g, h); resultado semelhante foi observado para teor de P na parte aérea (dados não apresentados), com o aumento da dose de P. De acordo com Lefebvre \& Glass (1982), a concentração de P na parte aérea teria papel central na regulação da taxa de absorção de $\mathrm{P}$ por unidade de raiz, de modo que os dois mecanismos sinalizadores, para manter a homeostase de P, seriam: um em nível celular e outro envolvendo múltiplos órgãos e, provavelmente, da parte aérea (Raghothama, 2000). De acordo com esse autor, em nível celular, o movimento de $\mathrm{P}$ inorgânico (Pi) para dentro e fora do vacúolo e a regulação do influxo e efluxo de $\mathrm{P}$ seriam os principais mecanismos para manter a homeostase de $\mathrm{P}$, enquanto a resposta relativa à planta inteira envolveria mecanismos mais complexos. Observa-se baixa EAP no solo LVd1, o que deve estar relacionado à sua maior capacidade de fixação de $\mathrm{P}$ em relação aos outros solos, evidenciada pelo menor valor de P-remanescente (Quadro 1). Andrade et al. (2000), avaliando o rendimento do feijoeiro em função do fornecimento ou da omissão de nutrientes em solos de várzea, observaram que o grau de limitação de $\mathrm{P}$ variou entre os solos o suficiente para reduzir a produção relativa de grãos a valores inferiores a $50 \%$ em relação ao tratamento completo, provavelmente em razão dos diferentes potenciais desses solos de liberar o P para as plantas. Segundo Fageria et al. (2004), a eficiência de recuperação do P pela planta do feijão é menor que $10 \%$, em solos de Cerrado, dependendo da dose aplicada. Em compensação, a eficiência de uso do $\mathrm{P}$ (produção de grãos por unidade de $\mathrm{P}$ acumulado na planta) é muito maior do que a de $\mathrm{N}$ e $\mathrm{K}$.

Foram observados aumentos progressivos e substanciais na eficiência de utilização de P (EUP), com o incremento das doses de $\mathrm{P}$ para todos os solos na condição não cultivada (Figura $2 \mathrm{e}, \mathrm{f}, \mathrm{g}, \mathrm{h}$ ). Esse comportamento mostra que em solo adubado pela primeira vez é muito mais forte a competição dele com a planta pelo $\mathrm{P}$ do fertilizante. Nessa situação, o acúmulo do nutriente pela planta é restringido (Figura 1e, f, g, h), e esta é forçada a otimizar o metabolismo, em função da quantidade que consegue absorver do nutriente. De acordo com Schachtman et al. (1998), quando o suprimento de P é limitado, as plantas crescem mais e as raízes aumentam a taxa de absorção, retranslocam $\mathrm{Pi}$ das folhas velhas e promovem uma depleção do Pi armazenado no vacúolo. Nota-se que, para a condição de solo já cultivado, quando a disponibilidade inicial desse nutriente no solo é mais favorável (Quadro 1) e o feijoeiro consegue absorver maior proporção do nutriente adicionado (Figura 1e, f, g, h), a EUP foi pouco alterada pelas doses de $\mathrm{P}$ (Figura 2e, f) ou ocorreu ausência de resposta à adição de doses crescentes de $\mathrm{P}$ (Figura $2 \mathrm{~g}, \mathrm{~h}$ ).

Num curto tempo, os fertilizantes fosfatados, quando aplicados, são mais eficientemente utilizados sobre solos com baixo teor de P, porém, em longo prazo, após significante quantidade de $\mathrm{P}$ ter sido adsorvida, ocorre equilíbrio com a dose de $\mathrm{P}$ aplicada; portanto, aplicações continuadas desse nutriente poderão ser utilizadas com similar eficiência de uso (Helyar, 1998). Nessa situação de adequado suprimento de P ocorre conversão do Pi absorvido e armazenamento deste em compostos orgânicos como ácido fítico (Schatchatman et al., 1998) e redução de absorção de Pi da solução (Bieleski \& Fergunson, 1983). Além disso, observase que, assim como ocorreu para a produção de MSPA (Figura 1a, b, c, d), os fatores relacionados ao solo, como textura e mineralogia, parecem sobrepor-se em relação àqueles relacionados à planta e ao histórico de uso do solo, de modo que solos com maior fator capacidade de $\mathrm{P}$, como os LVd, apresentaram certo ganho na EUP mesmo na condição de anteriormente manejados e adubados. Em se tratando da primeira adubação, a quantidade de $\mathrm{P}$ absorvida não foi suficiente para que a planta atingisse sua capacidade metabólica plena, visto que a EUP aumentou linearmente com as doses do nutriente na adubação.

Esses dados contrastam com os encontrados por Fageria (1998), para quem os valores de EUP geralmente diminuem com o aumento do fornecimento do nutriente para o feijoeiro, devido ao fato de haver nivelamento de produção, quando a disponibilidade de nutriente no sistema tende a ser alta, mas continua a ocorrer incremento em teor e conteúdo na planta. Assim, a eficiência de utilização de $\mathrm{P}$ é máxima em situação de menor disponibilidade e mínima em caso de elevada disponibilidade. Araújo (2000) afirma que 
a EUP pode contribuir para minimizar a restrição ao crescimento vegetal em condições de limitação no suprimento do nutriente. Esse aspecto é confirmado pelos resultados obtidos no presente trabalho, em que, na maior dose de $\mathrm{P}$ fornecida (480 $\mathrm{mg} \mathrm{dm}^{-3}$ de $\mathrm{P}$ ), a tendência de equiparação na produção de MSPA (Figura 1a, c, d) e também na produção de grãos (dados não apresentados) entre a condição cultivada (suprimento menos limitante) e a não cultivada (suprimento mais limitante) muito provavelmente foi condicionada pelas diferenças nos padrões de EUP nos dois casos (Figura 2e, f, g, h).

De acordo com os resultados, verifica-se que para os solos na condição cultivada, apesar de haver menor resposta à adição do nutriente em relação à condição não adubada anteriormente, uma dose menor de $\mathrm{P}$ tem efeito equivalente ao de altas doses em áreas não cultivadas; esse efeito é tão mais intenso quanto maior o tempo de cultivo da área, o que está ligado ao fato de que nessa condição o solo, além de apresentar maior quantidade de $\mathrm{P}$ disponível, compete menos com a planta pelo $\mathrm{P}$ adicionado, proporcionando, assim, melhor absorção do nutriente, mesmo com o fornecimento de menores doses. Isso sugere que a adubação fosfatada em solos de Cerrado está estreitamente relacionada com o histórico de uso do solo, sendo esse efeito maior ou menor em função das características físicas e mineralógicas do solo.

\section{CONCLUSÕES}

1. A adição de doses crescentes de $\mathrm{P}$ aos solos promove aumento dos componentes relacionados ao crescimento do feijoeiro, principalmente matéria seca e conteúdo de $\mathrm{P}$.

2. A condição de solo já cultivado potencializou as respostas do feijoeiro à adubação, sobretudo na menor dose de P. Nessa condição, houve maior absorção de P pelas plantas.

3. O histórico de uso do solo influencia tanto a absorção quanto a eficiência de utilização de $\mathrm{P}$ pelas plantas. No entanto, as condições que promovem maior absorção são opostas àquelas que permitem maior eficiência de utilização do nutriente, sobretudo nas condições de solo já cultivado.

4. A disponibilidade de $\mathrm{P}$ no solo foi mais dependente das características físicas, químicas e mineralógicas do que do histórico de uso do solo.

\section{AGRADECIMENTO}

À Fundação de Amparo à Pesquisa do Estado de Minas Gerais (FAPEMIG), pelo apoio financeiro concedido ao desenvolvimento deste trabalho.

\section{LITERATURA CITADA}

ANDRADE, C.A.B.; FAQUIN, V.; FURTINI NETO, A.E.; CURI, N.; VEIGA, P.M.R. \& ANDRADE, M.J.B. Fertilidade de solos de várzea do sul de minas gerais para o cultivo do feijoeiro. Pesq. Agropec. Bras., 35:2287-2294, 2000.

ARAÚJO, A.P. Eficiência vegetal de absorção e utilização de fósforo, com especial referência ao feijoeiro. In: NOVAIS, R.F.; ALVAREZ V., V.H. \& SCHAEFER, C.E.G.R. Tópicos em ciência do solo. Viçosa, MG, Sociedade Brasiléia de Ciência do Solo, 2000. p.163-212.

BALIGAR, V.C.; DUNCAN, R.R. \& FAGERIA, N.K. Soil plant interaction on nutrient use efficiency in plants: An overview. In: BALIGAR, V.C. \& DUNCAN, R.R. Crops as enhancers of nutrient use. San Diego, Academic, 1990. p.351-373.

BIELESKI, R.L. \& FERGUSON, I.B. Physiology and metabolism of phosphate and its compounds. In: LAUCHLI, A. \& BIELESKI, R.L., eds. Inorganic plant nutrition. New York, Springer-Verlag, 1983. p.422-449.

BOLLAND, M.D.A. \& GLIKES, R.J. The chemistry and agronomic effectiveness of phosphate fertilizers. J. Crop Produc., 1:139-163, 1998.

CARVALHO, A.M.; FAGERIA, N.K.; OLIVEIRA, I.P. \& KINJO, T. Resposta do feijoeiro à aplicação de fósforo em solos dos cerrados. R. Bras. Ci. Solo, 19:61-67, 1995.

CHAPUIS-LARDY, L.; BROSSARD, M. \& QUIQUAMPOIX, H. Assessing organic phosphorus status of Cerrado oxisols (Brazil) using 31P-NMR spectroscopy and phosphomonoesterase activity measurement. Canadian J. Soil Sci., 81:591-601, 2001.

COMISSÃO DE FERTILIDADE DO SOLO DO ESTADO DE MINAS GERAIS - CFSEMG. Recomendação para o uso de corretivos e fertilizantes em Minas Gerais: $5^{\circ}$ Aproximação. Viçosa, MG, 1999. 359p.

CONDRON, L.M. \& TIESSEN. H. Interactions of organic phosphorus in terrestrial ecosystems. In: TURNER, B.L.; FROSSARD, E. \& BALDWIN, D.S., eds. Organic phosphorus in the environment. Wallingford, CAB International, 2005. p.295-308.

FAGERIA, N.K. Eficiência de uso de fósforo pelos genótipos de feijão. R. Bras. Eng. Agríc. Amb., 2:128-131, 1998.

FAGERIA, N.K.; BARBOSA FILHO, M.P. \& STONE, L.F. Nutrição de fósforo na produção de feijoeiro. In: YAMADA, T. \& ABDALA, S.R.S. Fósforo na agricultura brasileira. Piracicaba, Potafos, 2004. p.435-453.

GUGGENBERGER, G.; CHRISTENSEN B.T.; RUBÆK, G. \& $\mathrm{ZECH}, \mathrm{W}$. Land use and fertilization effects on $\mathrm{P}$ forms in two European soils: Resins extraction and 31P-NMR analysis. Europ. J. Soil Sci., 47:605-614, 1996.

HELYAR, K.R. Efficiency of nutrient utilization and sustaning soil fertility with particular reference to phosphorus. Field Crops Res., 56:187-195, 1998. 
KIMANI, J.M. \& DERERA, J. Combining ability analysis across environments for some traits in dry bean (Phaseolus vulgaris L.) under low and high soil phosphorus conditions. Euphytica, 166:1-13, 2009.

LEFEBVRE, D.D. \& GLASS, A.D.M. Regulation of phosphate influx in barbley roots: Effects of phosphate deprivation and reduction of influx with provision of orthophosphate. Plant Physiol., 54:199-206, 1982.

MALAVOLTA, E.; VITTI, G.C. \& OLIVEIRA, S.A. Avaliação do estado nutricional das plantas: Princípios e aplicações. 2.ed. Piracicaba, Potafos, 1997. 319p.

MATOS, E.S.; MENDONÇA, E.S.; VILLANI, E.M.A.; LEITE, L.F.C. \& GALVÃO, J.C.C. Formas de fósforo no solo em sistemas de milho exclusivo e consorciado com feijão sob adubação orgânica e mineral. R. Bras. Ci. Solo, 30:625 $632,2006$.

MIRANDA, L.N.; AZEVEDO, J.A.; MIRANDA, J.C.C. \& GOMES, A.C. Produtividade do feijoeiro em resposta a adubação fosfatada e a regimes de irrigação em solo de cerrado. Pesq. Agropec. Bras., 35:703-710, 2000.

NOVAIS, R.F. \& SMYTH, T.J. Fósforo em solos e planta em condições tropicais. Viçosa, MG, Universidade Federal de Viçosa, 1999. 399p.

NOVAIS, R.F. \& MELLO, J.W.V. Relação solo-planta. In: NOVAIS, R.F.; ALVAREZ V., V.H.; BARROS, N.F.; FONTES, R.L.F.; CANTARUTTI, R.B. \& NEVES, J.C.L. Fertilidade do solo. Viçosa, MG, Sociedade Brasileira de Ciência do Solo, 2007. p.134-177.

NZIGUHEBA, G. \& BUNEMANN, E.K. Organic phosphorus dynamics in tropical agroecosystems. In: TURNER, B.L.; FROSSARD, E. \& BALDWIN, D.S., eds. Organic phosphorus in the environment. Cambridge, CABI Publishing, 2005. p.243-268.

PASTORINI, L.H.; BACARIN, M.A.; LOPES, N.F. \& LIMA, M.G.S. Crescimento inicial de feijoeiro submetido a diferentes doses de fósforo em solução nutritiva. R. Ceres, 47:219-228, 2000.
RAGHOTHAMA, K.G. Phosphate transport and signaling. Cur. Opin. Plant Biol.,3:182-187, 2000.

RAIJ, B.van. Fertilidade do solo e adubação. Piracicaba, Agronômica Ceres, 1991. 343p.

SÁ, J.C.M. Adubação fosfatada no sistema plantio direto. In: YAMADA, T. \& ABDALA, S.R.S. Fósforo na agricultura brasileira. Piracicaba, Potafos, 2004. p.201-220

SANTOS, D.R.; GATIBONI, L.C. \& KAMINSKI, J. Fatores que afetam a disponibilidade do fósforo e o manejo da adubação fosfatada em solos sob sistema plantio direto. Ci. Rural, 38:576-586, 2008.

SANTOS, H.P. \& TOMM, G.O. Disponibilidade de nutrientes e teor de matéria orgânica em função de sistemas de cultivo e de manejo de solo. Ci. Rural, 33:477-486, 2003.

SCHACHTAMAN, D.P.; REID, R.J. \& AYLING, S.M. Phosphorus uptake by plant: From soil to cell. Plant Physiol., 116:447-453, 1998.

SIDDIQI, M.Y. \& GLASS, A.D.M. Utilization index: a modified approach to the estimation and comparison of nutrient utilization efficiency in plants. J. Plant Nutr., 4:289-302, 1981.

VINCENT, A.G.; TURNERB, B.L. \& TANNER, E.V.J. Soil organic phosphorus dynamics following perturbation of litter cycling in a tropical moist forest. Europ. J. Soil Sci., 61:48-57, 2010.

WRIGHT, A.L. Soil phosphorus stocks and distribution in chemical fractions for long-term sugarcane, pasture, turfgrass, and forest systems in Florida. Nutr. Cycling Agroec., 83:223-231, 2009.

XAVIER, F.A.; ALMEIDA, E.F. \& IRENE, M.C. \& MENDONÇA, E.S. Soil phosphorus distribution in sequentially extracted fractions in tropical coffee-agroecosystems in the Atlantic Forest biome, Southeastern Brazil. Nutr. Cycl. Agroecosyst., 89:31-34, 2010. 